\title{
La recherche dans les universités de l'Ouest
}

Liste des thèses et des mémoires de masters soutenus au cours de l'année 2010

\section{(2) OpenEdition}

Journals

Édition électronique

URL : http://journals.openedition.org/abpo/2085

DOI : $10.4000 / a b p o .2085$

ISBN : 978-2-7535-1841-4

ISSN : 2108-6443

Éditeur

Presses universitaires de Rennes

\section{Édition imprimée}

Date de publication : 30 décembre 2011

Pagination : 213-226

ISBN : 978-2-7535-1839-1

ISSN : 0399-0826

\section{Référence électronique}

"La recherche dans les universités de l'Ouest », Annales de Bretagne et des Pays de l'Ouest [En ligne], 118-4 | 2011, mis en ligne le 30 décembre 2011, consulté le 19 mars 2021. URL : http:// journals.openedition.org/abpo/2085; DOI : https://doi.org/10.4000/abpo.2085

Ce document a été généré automatiquement le 19 mars 2021

(c) Presses universitaires de Rennes 


\section{La recherche dans les universités de l'Ouest}

Liste des thèses et des mémoires de masters soutenus au cours de l'année 2010

\section{Université d'Angers}

\section{Thèses}

1 El AMRANI, Frédérique née BOISSEAU, Jeunes filles des milieux populaires ruraux en Anjou dans la première moitié du XXe siècle, dir. C. BARD.

2 AVRANE, Colette, Genèse et application de la loi du 10 juillet 1915 ou le salaire minium des ouvrières à domicile, dir. C. BARD.

3 VÉLEZ, Anne, Histoire du sport féminin d'entre-deux-guerres en France, dir. C. B ARD.

\section{Master 2 (spécialité Recherche)}

4 BERLIN, Johanne, Biographie d'une féministe, Luce Sirkis, dir. C. BARD.

5 Bertolino Laugery, Carole, Les lettres de pardon du roi de France de 1567, dir. M. NASSIET.

6 BoteAu, Flavien, La France et l'Exposition Universelle de Montréal en 1967, dir. Y. DENÉCHÈRE.

7 BOURGEOIS, Lilian, L'histoire en chantant : de 1945 à nos jours, dir. C. BARD.

8 BRetAudeau, Angélique, La représentation des femmes dans le Mystères de la Passion d'Arnoul Gréban et de Jean Michel (xve siècle), dir. J-M. MATZ.

9 DREMEAUX, François, La présence française à Hong Kong dans l'entre-deux-guerres (1918-1941), dir. Y. DENÉCHÈRE.

10 GuESDON, Olivier, Sortir de la captivité, d'après les actes de chancellerie du consulat de France à Tunis entre 1680 et 1713 , dir. F. BRIZAY.

11 JUHEL, Enora, Le culte des saints dans le couvent franciscain observant de la Baumette à Angers, dir. J-M. MATZ. 
Juteau, Sébastien, Conscrits et société dans l'Anjou républicaine de 1868 à 1919, dir. A. LIGNEREUX.

MIANT, Justine, Entre solidarités et conflits : la société égyptienne au miroir du christianisme (début ve siècle), dir. J-Y. CARREZ-MARATRAY.

NuvilLE, Martin, Les infirmières Croix-Rouge pendant la guerre d'Indochine 1945-1954, dir. Y. DENÉCHÈRE.

ROULET, Gaultier, Les livres d'heures dans le diocèse d'Angers, dir. J-M. MATZ.

TAILLEFAIT, Romain, Le débat sur l'abolition de la peine de mort dans la presse française entre 1974 et 1981, dir. Y. DeNÉCHÈRE.

THIREAU, Camille, Administrer la révolution: les dysfonctionnements de l'administration du Maine-et-Loire de 1790 à 1799, dir. A. LignEREUX.

VAllaIS, Pierre, La paix dans l'Italie communale au XIIIe siècle. Analyse comparée des chroniques de Salimbene de Adam et de Rolandino de Padoue, dir. J-M. MATZ.

\section{Master 1(sont répertoriés ceux qui ont été déposés à la bibliothèque d'Angers)}

ALBERT, Thibault, L'iconoclasme politique. La place des symboles dans la légitimation du pouvoir sous la Restauration (1814-1830), dir. A. LIGNEREUX.

Assati, Alison, De l'élaboration à la publication, les inventaires des fonds anciens aux Archives départementales de Maine-et-Loire, dir. P. MARCILLOUX.

BaDos, Geoffrey, L'utilisation des pierres dans le livre XXXVII de l'Histoire Naturelle de Pline l'Ancien, dir. P. GaILlaRD-SeuX.

2 BARON, Anaïs, Le rôle de Konrad de Thuringe dans l'ordre teutonique (1233-1241), dir. M- M. de CEVINS.

Bellamy, Justine, Les archives du clergé régulier antérieures à 1790 : collecte, traitement et valorisation. $L a$ série $H$ des archives départementales de Maine-et-Loire, dir. P. MARCILLOUX.

BERTIN, Grégoire, Le cinéma dans les relations franco-américaines au regard des ambassadeurs en poste à Washington (1927-1942), dir. Y. DENÉcHÈRE.

BOURMAUD, Angélique, Les gardiens de la mémoire vendéenne: les archivistes départementaux, du lendemain des guerres de Vendée à la veille de la seconde guerre mondiale, dir. P. MARCILLOUX.

BOUVET, Joseph, L'Abbaye Saint-Serge d'Angers, première moitié du XIIe siècle, un centre culturel, dir. N-Y. TONNERRE.

BRIANT, Loïck, Les Filles du MRJC. L'exemple de la section des Pays de la Loire, dir. C. Bard.

CABANETOS, Damien, Les axes de circulation transpyrénéens, dir. Y. DENÉCHÈRE.

CABON, Marie, Introduction à l'étude de la rémission au XVIIIe siècle dans le ressort du présidial de Rennes, dir. M. NASSIET.

CAILLEAU, Amandine, La culture cinématographique et radiophonique du journal féministe La Française dans les années 1930, dir. C. BARD.

1 CARRÉ, Élodie, Cléopatre II et Cléopatre III, dir. J.-Y. CARREZ-MARATRAY. 
CHARDRON, Jean-François, La Chouannerie dans le district de Segré. De son acte de naissance manqué à la Paix de la Mabilais 1793-1795, dir. A. LIGNEREUX.

CHeSneAu, Marie, Les juges des enfants dans les tribunaux du ressort de la cour d'Appel d'Angers 1945-1957, dir. E. PIERRE.

CHÈVRE, François, Les "minériades" de juin 1990 à Bucarest vues par Le Monde et L'Humanité, dir. Y. DENÉCHÈRE.

Cosson, Julie, Défense du protestantisme français par un contemporain de la révocation de l'Edit de Nantes : vie et œuvres d'Elie Benoist, dir. D. BoIsson.

DEMARTY, Nadège, Les archives administratives d'Ancien Régime : de la collecte à la valorisation (1789-2010), l'exemple des séries $C$ et $D$ des archives départementales du Finistère, dir. P. MARCILLOUX.

DieumegARD, Camille, L'implication des laïcs dans le mariage catholique de Vatican II à aujourd'hui à travers deux paroisses d'Angers, dir. P. QUINCY-LEFÈBVRE.

DORVAUX, Karine, Françoise Gaspard, actrice et observatrice du féminisme politique, de 1977 à 1995, dir. C. BARD.

DRouET, Armand, Ali Silem et Dalila Morsly. Biographie d'un couple féministe d'origine algérienne, dir. C. BARD.

ÉMERIAU, François, Les Vendéens de Chaudron-en-Mauges à travers leurs dossiers de demande de pension, dir. A. LIGNEREUX.

FREUlon, Julie, Le recrutement des clercs majeurs en Anjou à la fin du xve siècle, dir. J.M. MATZ.

FREVAL, Lise, L'éducation surveillée au Maroc de la fin des années 1940 à la fin des années 1960 à travers les archives d'André Heinrich, dir. E. PIERRE.

GuERREIRO, Lucy, La noblesse en Anjou d'après les montres militaires de 1470, dir. J.-M. MATZ.

GuILlaRD, Justine, Le féminisme de Fiammetta Venner, dir. C. BARD.

GuILLET, Marlène, La Française et les colonies (1931-1939), dir. C. BARD.

HoF, Flory-Jan, Les partis politiques de droite en Afrique du sud au regard de la diplomatie française (1930-1948), dir. Y. DENÉCHÈRE.

JAUnAY, Julian, Les politiques d'édition de documents d'archives en Sarthe au XIXe siècle, dir. P. MARCILLOUX.

8 Kaltoumi, Assia, De la collecte à la valorisation: les fonds contemporains d'architectes depuis 1987. Le cas du Maine-et-Loire, dir. P. MARCILLOUX.

LECRU, Graziella, Le corps féminin à travers le journal La Française dans les années trente, dir. C. BARD.

LEZE, Thibault, La perception des services d'archives par leurs publics en Sarthe entre 1947 et 2010, dir. P. MARCILLOUX.

5 LIGER, Matthieu, Louis XI et l'Anjou. Essai sur une reprise en main, d'après les lettres du roi 1461-1483, dir. J.-M. MATZ.

2 LOUVET, Sylvain, La statutaire publique commémorative de 1800 à 1944. L'exemple de Rennes, dir. E. PIERRE.

MEIGNAN, Jean-Sébastien, Le règne du roi Lagide Ptolémée VI, dir. J.-Y. CARREZ-MARATRAY. 
MONEUZE, Nicolas, Les services éducatifs d'archives: le cas du service éducatif des archives départementales de la Sarthe; de son élaboration à nos jours, dir. P. MARCILLoux.

Mouton, Julie, Les moyens de revendication des femmes autochtones par le biais de leurs associations, dir. E. PIERRE.

PAPILLON, Lucie, Le féminisme d'Éliane Viennot dans les années 1970 et 1980, dir. C. BARD.

PASQUIER, Jérome, Le journal d'Auguste Charrier, soldat (1916-1917), dir. Y. DENÉCHÈRE.

PIEL, Denis, Le pouvoir de Foulque II Le Bon, comte d'Anjou de 941 à 960 : étude sur la puissance angevine au milieu du xe siècle, dir. N.-Y. TONNERRE.

PRÉCIGouT, Élise, L'enseignement ménager au travers des films fixes, dans l'enseignement primaire, des années 1950 aux années 1970, dir. P. QUINCY-LEFÈBVRE.

RIVA ROVEDA, Fabrice, Les relations internationales dans le domaine des archives. La place de l'archivistique italienne sur la scène internationale de 1910 à nos jours, dir. B. GRAILLES.

RoINARD, Anaël, La Constitution de la logique classificatoire d'un fonds d'archives diplomatiques: le cas de l'ambassade de France «Rome Saint-Siège » de 1793 à nos jours, dir. B. GRAILLES.

SAVARY, Carine, Les religieux et leurs livres en Anjou à la fin du XVIIIe siècle, dir. V. SARRAZIN.

SAVATIER, Tiffanie, Les archives hospitalières, de la conservation à la valorisation : l'exemple des archives départementales de Maine-et-Loire, dir. P. MARCILLOUX.

Schultz, Beverley, La fiabilité des Ethnika d'Étienne de Bysance pour la Macédoine, dir. E. PARMENTIER.

SÉJOURNÉ, Isabelle, Pierre-Honoré Chadaigne, dir. M. CAFFORT.

SÉVERIN, Marine, Le Dromos. Recherches en archéologie grecque et égyptienne (époques tardives), dir. J.-Y. CARREZ-MARATRAY.

TESSIER, Mélanie, La propagande de Vichy en Argentine de 1940 à 1944, dir. Y. DENÉCHÈRE.

THÉGNER, Émilie, Conflits et sociabilité à Durtal en 1668, dir. M. NASSIET.

VAUDRon, Gaëtan, Les désastres de la guerre de cent ans dans le Maine, d'après l'étude du compte du receveur de l'évêque de Mans, dir. J.-M. MATz.

VAUgondy, Steeve, La prohibition et le crime organisé aux États-Unis sous le regard de l'ambassadeur Paul Claudel : 1926-1933, dir. Y. DENÉCHÈRE.

1 VelLA, Renaud, L'assimilation des autochtones par les pensionnats du Québec au xxe siècle, dir. E. PIERRE.

72 Wolf, Guillermo, Des brebis noires créent Sud-PTT. Les femmes dans la construction $d u$ syndicat Sud PTT (1988-1994), dir. C. BARD.

\section{Université de Bretagne occidentale (Brest)}

\section{Thèses}

LE BOULANGER, Isabelle, L'abandon des enfants: pratiques, modalités, enjeux. L'exemple des Côtes du Nord au XIXe siècle (1811-1904), dir. C. BOUGEARD.

LE SEAC'H, Emmanuelle, Les ateliers de sculpteurs sur pierre en Basse Bretagne du Xve au XVIIe siècle, dir. F. ROUDAUT. 

politique, dir. F. BOUTHILLON.

\section{Master 2 (seuls les masters 2 sont répertoriées)}

AlEXANDRE, Gaëlle, Les massacres de Jaffa, dir. F. BOUTHILlon.

ANDRÉ, Mickael, Sur les traces de la Boudeuse et de l'Astrolabe, les premiers enquêteurs de La Pérouse, dir. P. POURCHASSE.

BARTHelme, Karine, Le mythe La Pérouse, dir. P. POURCHASSE.

BouIn, Alexandre, Les luttes paysannes dans les années 60 dans le Finistère, dir. C. BOUGEARD.

BRIAND, Émilie, Culte et utilisation de l'image d'Ernesto «Che » Guevarra, dir. F. BOUTHILLON.

CAN, Florian, Carlo Rosselli. De l'Italie à l'hérésie, dir. F. BOUTHILlon.

Colliou, Charlotte, Ar Men et la création d'une identité bretonne (1986-2010), dir. Y. TRANVOUEZ.

CORVISIER, Stéphane, La notion de décadence romaine chez les auteurs français du XVe au XXe siècle, dir. B. LANçON.

DeliLLE, Claire, La bande dessinée "L'espiègle Lili» : entre conformisme et contestation de 1909 à 1974, dir. Y. TRANVOUEZ

DURAND, Patricia, Assister ou réprimer la mendicité et le vagabondage ou « comment rendre les mendiants utiles à l'Etat sans les rendre malheureux ». L'exemple de la province de Bretagne au XVIIIe siècle, dir. P. JARNOUX.

Fichou, Marine, La famille de Rohan dans le fonds des Blancs-Manteaux, dir. Y. COATIVY

FlAMENT, Gwendal, Le 19e régiment d'infanterie de Brest durant la Première Guerre Mondiale, dir. Y. TRANVOUEZ

GAONACH, Marie, Colin de Tours, receveur de Champtoceaux (1350-1377), dir. Y. COATIVY.

GIDROL, Marc, Évolution sociale des quartiers de logements sociaux de Brest de 1950 à 1994, dir. C. BOUGEARD.

Guegan, Isabelle, Un drame né de la mer. Une épidémie de typhus à Brest et en Bretagne (1757-1758), dir. P. JARNOUX.

GuIAVARC'H, Yohann, Les « Castors » du Finistère, dir. C. BOUGEARD.

KERJEAN, Éric, La chute de l'amiral Kanaris. L'espion de Hitler et la résistance allemande, 1942-1945, dir. F. BOUTHILLON.

KERNEIS, Maiwenn, Normalisation linguistique et intégration scolaire: le défi de la politique linguistique catalane dans une société plurilingue, dir. Y. TRANVOUEZ.

LE BOURHIS, Jonathan, La désertion des ouvriers conscrits à l'arsenal de Brest sous le Premier Empire (an XII-1809), dir. P. POURCHASSE.

LEGRAND, Nicolas, Étude sociologique et géographique de la Résistance dans le Finistère, 1940-1944, dir. C. BOUGEARD.

LEMEILlAT, Marjolaine, Les actes de Jean Ier, duc de Bretagne (1237-1286), dir. Y. COATIVY.

LERICHE, Claire, Les douaires des princesses bretonnes du XIIIe au XVIe siècle, dir. Y. COATIVY. 


\section{Master 1} COATIVY. Y. CARLUER.

\section{Thèses} G. LE BOUËDEC.

\section{Master 2} BOUËDEC. S. LLINARES.

MORLON, Anne-Charlotte, Jeanne de Penthièvre, duchesse de Bretagne (1341-1364), dir. Y.

MORVAN, Émilie, Le dossier hagiographique de Saint Magloire : texte, traduction, commentaire, dir. M. COUMERT.

PETIT, Brendan, Sacré, martyr et sacrifice en Irlande de l'insurrection de Pâques à la guerre civile : apogée et mutilation du rêve irlandais, dir. F. BOUTHILLON.

SEIVERT, Kevin, Les Bas Bretons et la carrière des armes sous la IIIe République, dir. J.-

TANGUY, Camille, Instruction des femmes et des filles dans le Finistère entre 1879 et 1914 à travers la figure de l'institutrice publique, dir. Y. TRANVOUEZ.

VERGNAUD, Grégory, La France et l'ONU sous la IVe République, dir. D. LE GUYADER.

\section{Université de Bretagne Sud (Lorient)}

BOISSON, Pascal, Émile Marcesche, une trajectoire entrepreneuriale lorientaise (1868-1939), dir.

BASSENNE, Bertrand, Mémoire de l'esclavage et de la traite négrière en Sénégambie (1965-2007), dialectique de la diversité mémorielle, dir. O. Petré-Grenouilleau.

LAVILLE, Aymeric, L'arsenal de Lorient pendant l'Entre-deux-guerres (1927-1939), dir. G. LE

LECHAT, François, La géographie des trafics maritimes du Port de Redon au XVIIIe siècle, 2010, dir. G. LE BOUËDEC.

ÉGASSE, Benjamin, L'édification des ouvrages d'art défensifs de Lorient (1744-1796), dir.

DAVID-CARDENAZ, Marie-Ange, Les prénoms des Bretons aux XIVe et XVe siècles, dir. M. CASSET.

GILlOUARD, Audrey, Jonathan Williams Jr, un Américains dans le port de Nantes pendant la guerre d'Indépendance américaine, d'après les papers de Benjamin Franklin, dir. G. LE BOUËDEC.

HOUEIX, Yoann, Les relations entre Lorient et Cadix au XVIIIe siècle, dir. C. CÉRINO

MONTARNAL, Stéphanie, Les officiers mariniers de Brest de 1764 à 1775 : les origines, la carrière et la famille, dir. P. HRODEJ.

NAËL, Pierre-Marie, La démocratie municipale en temps de guerre: l'exemple du canton d'Auray pendant la Seconde Guerre mondiale, dir. L. LE GALL.

ROGUET, Myriam, Jean-Baptiste d'Après de Mannevillette en mer: le journal de navigation du Montaran (1753-1755), la route de l'Inde et de la Chine, dir. S. LLINARES. 
BOUDVIN, Sandrine, L'information maritime dans la presse écrite locale lorientaise du Premier Empire au début du Second Empire, dir. S. LLINARES.

RICHARD-MAUGAIN, Angélique, Le journal de navigation du Bailli de Suffren navire de la $3 e$ Compagnie des Indes (1788-1791), dir. S. LLINARES.

ALLAIN, Vincent, A. de La Borderie, un historien du XIXe siècle au service de la glorification du Moyen Âge breton, dir. J. QUAGHEBEUR.

HODICQ, Paul, L'évêché de Cornouaille au XIV e siècle, jeux de pouvoirs et devenirs, dir. J. QUAGHEBEUR.

MAUVAIS, Marion, Les rentiers de Vannes (1455-1458) : transcription dir. M. CASSET.

\section{Université de La Rochelle}

\section{Thèses}

SOUIAH, Karim, La mise en scène du patrimoine. Évolution des politiques de mise en valeur du patrimoine en Charente-Maritime (1830-1976), dir. G. MARTINIÈRE. OULD MOHAMED, Sidi Mohamed, L'évolution de la pêche en Mauritanie depuis l'indépendance à nos jours (1960-2009), dir. G. MARTINIÈRE.

\section{Master 2 (Recherche)}

BONIN, Sophie, Immigration des huguenots en Irlande pendant et après la révocation de l'Édit de Nantes, dir. D. POTON.

CAUWET, Estelle, Les femmes sous l'occupation : l'exemple de La Rochelle, dir. D. РОтоN.

CHAMPAGNE, Noémie, Stephen Girard et ses réseaux : l'art de tisser la toile, dir. L. VIDAL.

CHESNEL, André, Contacts entre Tziganes et sédentaires en France et dans l'Ouest de l'Europe (xve-XxIe siècles), dir. C. ILLOUZ. DASSONVAL, Amandine, Économie et société de La Rochelle au milieu du xve siècle à travers le registre d'Étienne Noirault, dir. M. BOCHACA.

7 LANVIN, Fabrice, La Rochelle et la grande pêche en Amérique du Nord au xVIIIe siècle (1713-1789), dir. D. POTON. au XIXe siècle en Charente-Maritime, dir. D. POTON. MOULINIER, Henri, 45 ans après sa création - Villeneuve les Salines: une ZUP «humanisée en priorité»? (1966-2010), dir. D. POTON.

130 PLANçon, Thomas, Le routier de la mer jusques au fleuve de Jourdain. Nouvellement imprimé à Rouen. Édition critique de l'édition publiée par Jacques le Forestier (Rouen v.1502-1510), dir. M. BOCHACA.

131 RIDEAU, Sophie, La représentation maritime dans la peinture des primitifs flamands, de la mer objet à la mer sujet. Étude comparative avec l'art des miniatures des manuscrits français, XIIIeXVe siècles, dir. P. PRÉTOU. SIM, Gérald, Le réseau consulaire français aux États-Unis (1815 - fin XIXe siècle), dir. L. VIDAL. 
VAUTRIN, Jean-Christophe, Histoire et mémoire de la shoah en Charente-maritime: les premières mesures antisémites d'État: recensements et statut des juifs (septembre 1940-mai 1941), dir. D. POTON.

\section{Université du Maine (Le Mans)}

\section{Thèses}

DOUMERC, François, Essai de reconstitution d'un espace princier : l'exemple des Ragonides dans le royaume franc puis dans le royaume de France et ses marges (vers 600-vers 1060), dir. A. RENOUX.

FERRAIOLI, Fernando, L'hecatostys: analisi della documentazione (L'hékatostys. Analyse de la documentation), dir. M. POLITO (Université de Salerne) et A. AVRAM. LARIDAN, Mathieu, L'intégration des étrangers en Gaule narbonnaise, dir. : R. SOUSSIGNAN. PAVÉ, François, Le péril jaune à la fin du XIXe siècle, fantasme ou inquiétude légitime?, dir. N. VIVIER.

138 TERRISSE, Marc, Les musées de sites archéologiques appréhendés en tant que vecteurs de développement local à travers trois études de cas préfigurant la mise en valeur opérationnelle du site de Chellah, dir. N. VIVIER.

VANTARD, Amélie, Les vocations pour les missions ad gentes (France, 1650-1850), dir. F. PITOU.

\section{Master 2}

AMBEZA, Carole, Culture savante et construction de l'État en France (1300-1380) : état de la question, dir. A. RENOUX. AUBERT, Anne-Lise, La fraude des aides au XVIIIe siècle avec l'exemple de la Région d'Amboise, dir. B. MUSSET. BERTIN, Éric, Les dominicains français et l'Amérique latine des années 1950 à nos jours, dir. D. AVON, D. PELLETIER.

CHAMBRIER, Aurélie, Les inondations de la Sarthe XIXe et XXe siècle, dir. N. VIVIER. CONSTANT, Philippe, La critique de jazz en France, bibliographie et sources commentées, dir. D. AVON. COUTIN, Fabien, Les manifestations séditieuses en Mayenne, 1830 -1880, dir. S. TISON. FEURER, Ludivine, Mécénat et politique sous Henri VIII, dir. C. MICHON.

GUILloteAu, Isabelle, Du paganisme au christianisme: un changement pour la femme scandinave ?, dir. A. RENOUX.

HERBÉ, Eudes-Emmanuel, Mottes et maisons fortes dans le canton d'Authon du Perche, XIe-XVIe siècles, dir. A. RENOUX.

HULIN, Émile, L'environnement médiéval de Castelnau-Magnoac vu à travers une charte de 1465, dir. E. JOHANS, V. CORRIOL. JACQUIER, Corinne, Les gens de métier au Mans, dir. S. GRANGER.

151 JOLIVET, Marjolaine, L'alimentation des moines de l'Abbaye de Perseigne, dir. B. MUSSET. 
LEPOTIER, François, Histoire d'une pratique médicale: l'électro-encéphalographie. France des années 1930 aux années 1960, dir. H. GUILLEMAIN.

LETESSIER, Virginie, Le collège royal de La Flèche 1764-1776, dir. L. BOURQUIN.

Limoes, Amalia, La sculpture ornementale dans l'abbaye Notre Dame de l'Épine (Évron, Mayenne), xe-xve siècles, dir. A. RENOUX.

NOLAND, Thomas, La religion dans la musique: EU, France, Sénégal (rap et rock), dir. D. AVON.

OUCHTAR, Karim, Habitats nobles maçonnés dans les cantons du Mans, XIVe-XVIe siècles, dir. A. RENOUX.

PoIsson, Manon, Claude Haton, le protestantisme et les protestants : le regard d'un prêtre sur la diffusion de la Réforme en France (1553-1561), dir. L. BOURQUIN.

POLARD, André, Écrire l'histoire de l'épilepsie, dir. H. GUILLEMAIN.

TANSORIER, Sarah, La perception de la religion gauloise dans la bande dessinée Astérix, dir. A. ALLELY.

TOSTAIN, Virginie, Le mobilier des églises à la fin du Moyen Âge : le cas des églises argentanaises au Xve siècle d'après les sources comptables, dir. A. RENOUX.

\section{Master 1}

1 ABARREAU, Nathalie, Le mobilier mérovingien en Sarthe : état des lieux, dir. A. RENOUX.

BESNIER, David, L'assistance et la lutte contre la mendicité et le vagabondage à Laval sous l'Ancien Régime (1658-1797), dir. F. PITou.

BOIRON, Vincent, Le logement insalubre au Mans, 1832-1902, dir. N. VIVIER.

BRILLOUET, Jéromine: Les Grands magasins au Mans, 1887-1926. "Temple élevé à la folie dépensière de la mode ", dir. N. VIVIER.

CHARPENTIER, Alizée, Deux petits monastères féminins du sud Maine à la fin de l'Ancien Régime, dir. S. GRANGER.

CHEVALLIER, Noémie, Le chapitre cathédral de Sées au XVIIIe siècle, dir. S. GRANGER.

GUÉRIN, Laurence, Le patrimoine hospitalier et sanitaire en Pays de Loire, dir. H GUILLEMAIN.

Joyaux, Mathieu, Hervé Bazin et la folie, images de la psychiatrie en France (1930-1960), dir. H. GUILLEMAIN.

LAVAUD, Charles, Le poilu chante sa réalité: les chansons des journaux de tranchées en 1917, dir. S. TISON.

LECONTE, Zoé, La vision du travail des femmes dans les chemins de fer français entre 1885 et 1914, dir. N. VIVIER.

LEDOUX, Alexandre, Auberges et aubergistes au Mans, rive droite, XVIIIe siècle, dir. S. GRANGER.

LoNGIN, Martine, La représentation du Diable dans les églises de la Sarthe (xIIe-Xvie siècle), dir. A. RENOUX.

ZIMMER, Karl, Débats et perceptions : l'islam au miroir du Secrétariat Unifié de la Quatrième Internationale, dir. D. AVON. 


\section{Université de Nantes}

\section{Thèses}

BOSSARD, Stéphane, Les relations sino-indiennes, 1972-1988, dir. J. WEBER.

ChAIllou, Virginie, De l'Afrique orientale à l'océan Indien occidental. Histoire des engagés africains à la Réunion au XIXe siècle, dir. J. WEBER.

FOURNIER, Caroline, Les bains d'Al-Andalus : espaces, formes et fonctions (VIIIe-XVe siècle), dir. C. MAZZoli-Guintard.

LE Doze, Philippe, Le Parnasse face à l'olympe. De la poésie comme mode de communication politique à l'époque d'Octavien/Auguste, dir. F. HURLET.

Rus, Dana Maria, Mariages et remariages sur le territoire du 2e régiment roumain de frontière à l'époque moderne: perspectives démographiques et anthropologiques, dir. T. NICOARA et M. CATALA, cohabilitation Université Cluj Napoca

VeLE, Anna Maria, Les relations franco-roumaines, 1866-1914, dir. N. BocsAn et J.-P. BoIs, cohabilitation Université de Cluj Napoca.

VINCIGUERRA, Anthony, La matière arabe de l'alchimie latine. Étude du contexte de réception et de reconstruction de la science de la fabrication artificielle de l'or et de l'argent, milieu XIIemilieu xille. Une contribution à l'histoire des relations entre Orient et Occident au Moyen Âge, dir. J. TOLAN.

\section{Master 2 (Histoire et Histoire de l'art)}

ARCAS, Renaud, Penser la Stasis en Grèce ancienne, dir. S. Milanezi et I. PAPAdopoulou.

BARRE, Romain, L'iconographie des Antigonides (Antigone Le Borgne, Démétrios Poliorcete, Antigone Gonatas), dir. I. PImouguet-PedARRos.

Bonamy, Alice, Haiffa sous le mandat britanique : regards croisés : archives du poste de Haïa, historien-nes israélien-nes, palestien-nes, français-ses et témoignages oraux de palestien-nes, originaires de Haïfa, dir. B. SALVAING.

Bregeon-Henwood, Flore, Place et rôle des gravures de Jean-Baptiste Greuze de la collection Cacault au sein du musée école de Clisson, dir. H. RousteAU-CHAMBON.

Chauveau, Céline, Histoire et architecture du château de Montmirail (Sarthe) à l'époque médiévale, dir. N. FAUCHERRE.

6 CORREIRA, Alexandre, La politique économique du Bharatiya janata Party au pouvoir (1998-2004), dir. L. JALABERT.

FICHOU, Kilian, La France vue par la presse indienne (2007-2009), dir. B. joly.

FONTAINE, Vincent, Récits de voyage et cartographie en Afrique de l'Ouest à l'époque moderne, dir. A. DE ALMEIDA MENDES.

GARREAU, Alexis, Le domaine de Mesnard la Barotière de 1945 à 1967, dir. B. SALVAING.

GUERRY, Claudie, Le décor peint du pavillon des chemins de fer. Exposition internationale de 1937, dir. N. FAUCHERRE.

1 Guillon, David, La cérémonie du triomphe de 43 av. J.-C. à la mort d'Auguste, entre concurrence et politique dynastique, dir. F. HURLET. 
LE Mouel, Aanor, L'Islam dans l'imaginaire florantin du Trecento, dir. J. TolAn.

LEBONNOIS, Pierre-Emmanuel Les contingents barbares dans les armées héléniques. L'apport des barbares dans les pratiques guerrières des Grecs dans les domaines techniques et tactiles, dir. I. Pimouguet-PedARRos.

LEMAIRE, Yann, La section S.F.I.O. De la Montagne (1944-1958), dir. B. JOLY.

MAHE, Mevena, La dame de Shalott de J.W. Waterhouse (1888): une cuvre unique entre héritage anglais et innovation française, dir. A. BONNET.

MARTIN, Emmanuelle, Ernest Pignon-Ernest: parcours d'une reconnaissance, dir. N. FAUCHERRE.

MARY, Ainsline, Drusilla, sceur de Caligula, dir. F. HURLET.

MORICE, Julien, Al-ma'Mun (1185-1232), dir. J. Tolan.

NICOLLE, Élise, Terre de feu : reconnaissance et stratégie de 1520 à 1701 dir. M. ACERRA.

PERNET, Pauline, Une famille patricienne entre République et Empire, dir. F. HURLET.

PloQuin, Marion, Contribution à l'étude du faubourg Saint-Jacques de Nantes: histoire et architecture de l'église Saint-Jacques et de la tour de Pirmil, dir. N. FAUCHERRE.

RIALLAND, Yoann, La Mauritanie au xxe siècle : la transition politique entre la fin de la période colloniale et le début de l'indépendance (1958-1968), dir. B. SALVAING.

RICORDEAU, Hubert, Les politiques de la contre-révolution et l'organisation du pouvoir sur le territoire du Haut-Pérou durant de guerres d'indépendance, 1805-1825, dir. С. THIBAUD.

RIFFAULT, Angéline, L'exposition Ashes and snow et son monatic museum : étude des enjeux du projet artistique, dir. N. FAUCHERRE.

ROYER, Céline, Les Monti, l'évolution originale d'une famille de la noblesse de robe nantaise au XVIIe siècle, dir. G. SAUPIN.

TERRIEN, Nicolas, La course maritime et la naissance des nations dans l'Amérique espagnole (1512-1824) dir. C. THIBAUD.

TOULleLAN, Camille, la création du mythe polynésien dans et par l'art européenentre. Existe-il une spécificité polynésienne dans la représentation européenne de l'exotisme, dir. M. ACERRA et H. ROUSTEAU-CHAMBON.

VesCHAMBRE, Guillaume, Le voyage des officiels romains en Égypte d'Auguste à Diocletien, dir. F. HURLET.

VIDAL-Mothes, Thibault, La résistance des Parianos et des Pastusos à la République de Colombie, 1809-1829, dir. C. THIBAUD.

VILLERET, Maud, Le commerce alimentaire à Tours au XVIIe siècle dir. N. COQUERY.

Volz, Émilie, Chevalerie errante et pouvoir politique à la fin du Moyen-Age, dir. P. JOSSERAND.

VoIsIN, Etienne Le général de Gaulle et trois quotidiens nantais: Ouest-France, L'Éclair et La Résistance de l'Ouest, 1958-1962, dir. L. JALABERT.

\section{Master 1 (Histoire et Histoire de l'art)}

213 Amosse, Diane, l'Image d'Élisabeth 1re d'Angleterre, dir. G. SAUPIN.

BIGUET, Adrien, La coopération militaire Française en Amérique Centrale et plus particulièrement au Guatémala, Salvador et Nicaragua? A-t-il existé une politique Française de 
diffusion de la théorie militaire Française contre-révolutionnaire dans les trois pays Centraméricains de 1960 aux années 1980, dir. C. THIBAUD.

BOUlESTREAU, Marion, La mise en œuvre des politiques agricoles du New Deal vue à travers les photographies de la FSA (Farm Security Administration), dir. P. PERETZ.

216 Chaigne, Damien, L'activité de la chancellerie des Ducs de Bretagne pendant les règnes de jean IV et Jean V (1364-1442), dir. J.-L. SARRAZIN.

217 CheVReAu, Antoine, Les représentations du Groënland en France à l'époque moderne, dir. E. SCHNAKENBOURG.

218 DELIÈRE, Thomas, La représentation de l'Islam chez Guillaume de Tyr et ses continuateurs, dir. J. TOLAN.

DuBoIs, Marie, Cléopâtre VII philopatos, dir. S. MiLANEZI.

DufIEFF, Corentin, la reconstruction de Nantes : la période des baraquements (1943-1966), dir. L. JALABERT. Non consultable pendant 2 ans.

DuPouY, Pierre-André, L'Union Patriotique (mars 1984-avril 1987). Une expérience d'opposition légale et démocratique de la gauche radicale colombienne, dir. C. THIBAUD. Foucher, Marion, Étude des fusailloles de Chochula (Puebla, Mexique), dir. S. Boulud et M. BAJON.

GADET, Rémi, Le Parti Communiste français en Loire-Atlantique (1958-1981), dir. B. JoLY.

GARREAU, Quentin, Les relations franco-britaniques dans le sous-continent indien de 1939-1947, dir. J. WEBER.

GUIBERT, Alice, Le rôle des consulats français aux États-unis dans le commerce FrancoAméricain à la fin du XIIIe siècle, dir. G. SAUPIN.

Guillou, Adeline, La mise en défense de la ville de Nantes à la fin du Xvie siècle (1589-1598), dir. G. SAUPIN.

HERVÉ, Aurélie, Les peintres de marines français (XVIIIe-début XIXe siècle), dir. M. ACERRA.

HeRvouet, Mathieu, Entre survie et résistance, peuples indiens dans la Sierra Norte de Puebla de la fin des années 1980 à nos jours, dir. C. THIBAUD.

229 LE MoIng, Xavier, Contexte diplomatique de l'ambassade de Hasekura Tsunenaga (1613), dir. M. ACERRA.

230 LEMÉE, Hélène, Un voyageur archéologue à la découverte de la Lycie: Charles Fellows 1799-1860, dir. S. MiLANEZI.

231 LUCAS, Amélie, Les relations économiques entre l'Espagne et les Philippines au XVIIIe siècle, dir. G. SAUPIN.

MARCHAND, Nadia, La société coloniale sous le regard de trois voyageurs (2e moitié du XVIIIe siècle), dir. M. ACERRA.

MARO, Guillaume, Les relations Franco-Bulgares de 1975 à 1989, dir. L. JALABERT. Non consultable pendant 2 ans.

234 MARoger, Daphné, La céramique. Histoire et techniques du Néolithique au XVIIIe siècle, dir. L. CHAZALON.

MÉZIÈRES, Guillaume, Des radios en mouvements : histoire de la fédération nationale des radios libres (1981-1986), dir. B. JoLy. Non consultable pendant 2 ans 
PÉREZ, Olivier, Les Italiens-Américains de New York et l'Italie durant l'entre-deux-guerres (1921-1938), dir. P. PERETZ.

PIETRI, François-Xavier, Programmes militaires soviétiques issus de projets allemands (1945-1955), dir. B. Joly. Non consultable pendant 2 ans.

PONTGERARD, Florian-Benoît, Le réaménagement du quartier nantais du Marchix 1945-1976, dir. M. CATAlA. Non consultable pendant 2 ans.

Ribeiro, Nicolas, L'image de la Flibuste en Martinique de 1635 à 1664, dir. A. De AlmeidA MENDES et M. ACERRA.

RouINSARD, Morgane, Les voyages et ambassades de Ghillebert de Lannoy (1386-1462), dir. P. JOSSERAND.

241 ROYER, Ségolène, L'image des Russes et du monde russo-varege dans les sources byzantines (IXeXIe siècle), dir. N. DROCOURT.

ROUGE, Gaël, Les mouvements sociaux aux chantiers navals de Saint-Nazaire (1955-1968), dir. L. JALABERT.

TANGI, Maël, Historique de la maison des hommes et des techniques à Nantes et du collectif des associations du patrimoine industriel et portuaire nantais, dir. Guy SAUPIN. (1968-1974), dir. P. PERETZ.

\section{Université de Poitiers}

\section{Thèses}

BRUDY Pascale, 'Refectio' et 'meditatio'. Les réfectoires du Centre-Ouest, XIIe-XIIIe siècles, dir. C. TREFFORT.

DEHOUX Esther, Les saints guerriers Xe-XIIe siècles, dir. M. AURELL.

MASTORGIO Laurent, Les Creuzé: une famille châtelleraudaise entre 1600 et 1840. Aspects sociaux, économiques et culturels, dir. J. PÉRET.

Memin Bonnaud Muriel, Justice et société en Bressuirais à la fin du Xve siècle, dir. M. AURELL.

PEDRO Martinho, La persistance des autorités traditionnelles au Mozambique colonial (1834-1974), dir. F. CHAUVAUD.

\section{Master 2}

BONNET Anthony, La carrière nationale de Jean-Pierre Raffarin, 1974-2010, dir. J. GRÉVY.

CADET Amandine, Les consultations civiques de l'oracle de Claros à l'époque romaine, du règne de Trajan à celui de Septime Sévère, dir. Y. LAFOND.

COURRoux Pierre, Le merveilleux et l'histoire à la fin du Moyen Âge: l'exemple de Jean d'Outremeuse, dir. Е. Воzоку.

LE HENANFF-BERTOUX Cyril, L'élaboration de la mémoire résistante en Morvan : une construction complexe. L'exemple du maquis Bernard, 1944-2010, dir. J. GRÉVY. 
LuAULT Noémie, Territorialisation et colonisation entre Tarragone et Lérida au XIIe siècle, dir. S. BOISSELLIER.

MASSE Julien, Être gréviste dans le Poitou-Charentes entre 1880 et 1954, dir. F. CHAUVAUD.

Quillon Angèle, Traduction et commentaire du poème XLVI de Théodulf d'Orléans, dir. C. TREFFORT.

SCHERER Laurène, Starlettes des années 30. Quelle image féminine?, dir. F. CHAUVAUD.

ViGNERON Nelly, La sainteté féminine dans les drames de Hrotsvitha de Gandersheim, dir. E. BOZOKY.

\section{Master 1}

AUDRAIN Maël, Les rôles des tailles de la Chapelle-Montreuil : éclairage sur la situation socioéconomique d'une paroisse poitevine dans le premier tiers du XVIIIe siècle, dir. J. PÉRET.

AYRAULT Jennifer, Les affaires criminelles en Gâtine poitevine dans la seconde moitié du xVIIIe siècle d'après les procédures du greffe criminel du présidial de Poitiers, 1750-1790, dir. F. VIGIER.

Bodin Camille, Le féminin dans le divin : Aphrodite, dir. L. BODIoU.

BRAUD Alexandre, La seigneurie de Montmoreau dans la première moitié du XVIIIe siècle: prérogatives judiciaires, personnels et premières approches d'activités, dir. F. VIGIER.

CAPET Elodie, Les Plantagenêts et les cisterciens, dir. M. AURELL.

CATELEAu Julie, Gaia, déesse de la Terre, dir. L. Bodiou.

CHEVReUl Arthur, De Jennings à Deepwater Horizon : l'introduction de l'industrie pétrolière en Louisiane, dir. E. KOCHER-MARBOEUF.

Couloumy Jennifer, Le vol et les voleurs dans la Vienne au XIXe siècle, dir. F. CHAUVAUD.

DEMESLAY Benjamin, Bernard de Clairvaux entre texte et monde. Un écrivain total au XIIe siècle, dir. T. DESWARTE.

DeSMOULINS Gérard, Les réquisitions dans la Vienne durant l'Occupation, dir. E. KOCHER MARBOEUF.

FOURNIER Mickaël, La trahison dans la chronique d'Adémar de Chabannes, dir. M. SORIA.

GIRET Amélie, La mémoire maritime du fleuve Saint Laurent sous le régime français dans les fonds québecois, dir. T. SAUZEAU.

GRIS Séverine, Guerre d'indépendance ou guerre d'insurrection? Lecture et interprétation de la guerre d'Amérique à travers la presse française (1775-1785), dir. A. JOLLET.

KowALCZYK Cassiopée, La nuit à Paris à la fin du Moyen Âge, XIve-Xve siècles, dir. L. HABLOT.

LEPINÇON Léo, Les Républicains espagnols dans la Vienne, 1936-1940, dir. G. BOURGEOIS.

MADI NGABOU Nahoalou, La propriété paysanne à Saint-Sauvant de 1786 à 1796, dir. A. JOLLET.

Michaud Clément, Les relations franco-espagnoles lors de l'entrée de l'Espagne dans les communautés européennes à travers El Pais et le Figaro, dir. C. MANIGAND.

Michel Etienne, Les chars français dans la campagne de mai-juin 1940. Retour sur les évolutions et les échecs durant l'Entre Deux Guerre, dir. E. KOCHER-MARBOEUF. 
MOLINARI Hélène, La commémoration du bicentaire de l'autonomie de la Finlande en 2009, dir. S. JAHAN.

MOURREAU Anne-Gaëlle, La captivité des prisonniers français en Angleterre durant la guerre de Cent Ans, dir. L. HABLOT.

MUZARD Marie, Les images de l'adultère dans la presse satirique de la Belle Époque : l'exemple $d u$ Rire, dir. F. CHAUVAUD.

PERIES Marjorie, L'abbé Suger de Saint-Denis, la royauté et la papauté : relations, confrontations et dépendance 1106-1151, dir. T. DESWARTE.

PlANTEY Marie Anaïs, Mon et armoirie. Se dire et se mettre en scène dans les sociétés du Japon et de l'Europe médiéval, dir. L. HABLOT.

PRIEUR Lauriane, La paroisse d'Archigny dans la seconde moitié du XVIIIe siècle, 1750-1790, dir. J. PÉRET.

Ricou Marine, La faculté de droit de Poitiers et ses étudiants dans la société poitevine au début $d u$ XVIIe siècle, dir. D. TURREL.

RIFFAULT Élise, Des jeunes filles devenues femmes dans le roman grec ancien, dir. L. BODIOU.

ROUILLARD Stéphanie, La réception de la «Gargonne » dans l'Entre-deux-guerres, dir. J. GRÉVY.

SUBES Bruno, Un siècle d'histoire des prisons de Poitiers: les détenus de la maison d'arrêt de Poitiers entre 1848 et 1949, dir. F. CHAUVAUD.

THomas Hélène, La célébration des Bourbons dans les Deux Sèvres sous la Restauration, dir. G. MALANDAIN.

TORRES Hugo, La question esclavagiste dans un journal abolitionniste francophone de Louisiane durant la guerre de Sécession américaine (1861-1865) : idéologie et identités, dir. S. JAHAN.

\section{Université de Rennes 2}

\section{Thèses}

Groussard, David, La gestion de l'eau dans les villes bretonnes aux XVIIe et XVIIIe siècle, dir. S. BIANCHI.

SOUPRAYEN, Gérald, Sélection et Recrutement des Officiers semi-direct par l'École de Cadres de Rouffach à l'Ecole Militaire de Strasbourg (1945-1986), dir. V. JoLY.

1 CLÉRIVET, Marc, La Danse de tradition populaire en Haute-Bretagne: XIXe et XXe siècles, dir. G. NICOLAS.

CARDON-QUINT, Clémence, Les prises de position des professeurs de français face aux réformes et projets de réforme de leur discipline (1946-1989), dir. G. Nicolas.

Master (les mémoires suivants sont déposés à la bibliothèque du CERHIO)

AMBRoISE, Benoît, Saint-Brieuc pendant les guerres de la Ligue (1589-1598), dir. P. HAMON.

Belhomme, Vanessa, La Vie latine de saint Efflam : réédition, traduction, commentaires, dir. B. MERDRIGNAC.

BELLAT, Typhaine, Les monnaies de la vicomté de Limoges durant la domination bretonne (1275-1360) : la collection du Musée Dobrée à Nantes, dir. P.-Y. LAFFONT et G. SALAUN. 
BERTRAND, Sylvain, Le double portrait de Philippe Le Bon et de Charles de Charolais dans les enluminures et chroniques de la cour de Bourgogne: modalités et enjeux de la représentation dynastique du pouvoir, dir. F. MERCIER.

BILLAUD, Daniel, Église et société en Vendée sous l'Occupation, dir. M. BERGÈre.

BOsSÉ, Camille, Le mouvement étudiant durant la dictature en Argentine à partir du cas de La Plata (1976-1983), dir. L. CAPDEVILA.

BOULONNE, Pierre, Le mariage dans les pays de coutume : étude comparée des établissements en Nord Bretagne et Plaine de Caen au XVIIIe siècle, dir. A. ANTOINE.

BRANLANT, Aurélie, Écrire et lire en temps de guerre: le cas des soldats de l'Empire, dir. Y. LAGADEC.

BRICAULT, Valérie, Les boîtes à chef en Basse-Bretagne, dir. G. PRovost.

CHABEAUX, Tanguy, La bataille de Saint-Aubin-du-Cormier: de l'histoire à la mémoire. Reconstitution historiographique et mémorielle de la «Cruelle rencontre» (xve-XIxe siècle), dir. F. MERCIER et J. Le BiHAN.

CHAPUT, Paul, La France face à l'initiative de défense stratégique de Ronald Reagan (1983-1986) : de la guerre des étoiles à la construction européenne, dir. J. SAINCLIVIER.

CHAPY, Jean-Romain, L'éducation française laïque dans l'Empire Ottoman (1856-1914), dir. G. NICOLAS.

CHAUvot, Olivia, Le Parlement de Bretagne et le religieux à Rennes quelles relations ? : étude menée à travers les arrêts sur remontrance (1643-1720), dir. G. AUBERT et G. PROVOST.

DAVIAUD, Florian, Le district de Bain au début de la Révolution : les élus dans les échanges entre territoire et nation (1790-1794), dir. D. GODINEAU.

DECOMBE, Elfie, La délinquance féminine dans les Côtes-du-Nord sous la Révolution, dir.

D. GODINEAU.

DESCHAMPS, Tiphaine, L'aliment de l'Autre: confrontation et métissage des cultures alimentaires dans l'ouest du Mexique au XVIe et XVIIe siècle, dir. F. QUELLIER.

DESHAIS, Audrey, Les imprimés de la fin de l'Affaire de Bretagne (1770-1774) à partir des fonds rennais, dir. G. AUBERT.

ESPIGAT, Florent, Le soldat en images: l'iconographie des soldats français de la Révolution (1789-1799), dir. D. GODINEAU.

2 GAILLET, Thomas, "Des idées anarchiques... » : la surveillance des anarchistes en Ille-et-Vilaine de 1894 à 1914, dir. P. KARILA-COHEN.

3 GAONAC'H, Yann, L'eau et les hommes dans le marais de Dol: des structures d'Ancien Régime à l'association des propriétaires des marais de Dol (xvie siècle - milieu du xixe siècle), dir.

\section{A. ANTOINE.}

GARNIER, Thibaud, Promotion et développement de l'anglais dans l'okinawa d'après-guerre, dir. V. JOLY.

5 GODET, Émilie, Le mobilier métallique civil et domestique issu des communs et de la cuisine du château du Guildo (22), dir. P.-Y. LAFFONT.

KERRIEN, Fanny, Les affiches de propagande reflet des rivalités idéologiques et politiques de la mise en place des régimes totalitaires à la fin de la guerre, dir. J. SAINCLIVIER. 
317 LE HÔ, Tiphaine, Les marchands de toiles d'Uzel : l'ascension d'une bourgeoisie rurale (v.1750-v. 1850), dir. Y. LAGADEC.

LE MARCHAND, Yves, Lien imaginé, lien perçu : la relation père-fils au Moyen Âge. L'exemple de Guillaume le Conquérant et de ses fils (XIe-XIIe siècle), dir. F. MAZEL. LE MIGNANT, Brice, 1900-1996: un siècle de politique dans les jeux olympiques au prisme du "Ouest-France », dir. M. BERGÈRE. LIGIER, Martine, Dictature, transition démocratique et rock'n'roll en Argentine (1976-1985), dir. L. CAPDEVILA.

321 LOUESSARD, Stéphane, Jean Le Laboureur, sieur de Bléranval et sa "Relation du voyage de la Royne de Pologne»: la vision de la Pologne à l'arrivée de Louise Marie de Gonzague, dir. A. ANTOINE. MADEC, Cécile, Identité réunionnaise : identité française ?, dir. J.-F. TANGUY. MANCEA, Camelia Niculina, La position de la France relative à l'adhésion de la Roumanie à l'Union européenne: aspects politiques, diplomatiques, et socio-culturels (1995-2007), dir. E. DROIT. MOTTAIS, Alexandre, La construction de l'image du Sarrazin infidèle, de la période patristique à la première croisade (IVe-XIe siècle), dir. I. RosÉ. NONET, Emmanuelle, La maison Deyrolle : une version moderne du cabinet de curiosités?, dir. G. AUBERT.

PALLUEL, Annaïg, Normes de genre et représentations sociales à l'épreuve de la guerre : la société bolivienne dans la Guerre du Chaco (1932-1935), dir. L. CAPDEVILA et G. BoRRAS. PERRIER, Camille, Bâtiments franciscains de Haute Bretagne du Moyen Âge au XIXe siècle : état de la recherche et sources disponibles, dir. P.-Y. LAFFONT. PICHARD-RIVALAN, Mathieu, Pouvoir municipal et société à Rennes (1620-1630), dir. G. AUBERT et P. HAMON. PIERRE, Arnaud, La France en Espagne (1843-1848) : réalités et limites de l'influence française en Espagne, dir. L. CAPDEVILA. PIGEON, Élodie, La mémoire du Cardinal Wolsey à Hampton court et sa mémoire aujourd'hui, dir. J.-M. LE GALL. PLADYS, Clémentine, Étude des granges cisterciennes en Bretagne aux XIIe et XIIIe siècles: exemple des granges des abbayes de Saint-Aubin-du-Cormier et de Boquen, dir. F. MAZEL et B. MERDRIGNAC. POUESSEL, Karine, Rennes pendant la première guerre de Religion : de la gestion de la guerre, de la famine et de la pauvreté par le pouvoir municipal vers une capitale provinciale grâce à l'installation du Parlement, dir. P. HAMON. PRÉAULT, Clémence, L'habitat en Ille-et-Vilaine à la fin du XVIIIe siècle à partir des saisies révolutionnaires, dir. J.-P. LETHUILLIER. QUENTRIC, Sylvie, Des relations familiales chez les privilégiés à partir d'un échantillon de factums rennais (1re moitié du XVIIIe siècle), dir. E. SABLAYRolles. QUINTON, François, L'Europe vue de droite: au coeur du «Figaro magazine» (1978-1984), dir. G. RICHARD et J. SAINCLIVIER. 
RAMEL, Jean-Loup, La prédication médiévale à travers les réécritures hagiographiques bretonnes, dir. B. MERDRIGNAC.

REGEREAU, Anaïs, Les vestiges archéologiques des fours à chaux datant du XIXe siècle en Mayenne, dir. D. Ріснот.

REINBOLD, Aurélie, Le paysage en forêt de Rennes : eaux et forêts, incultes, dir. D. РICHOT.

RÉVILLON, Jérémy, La politique étrangère du Rwanda après le génocide: dans quelle mesure futelle influencée par celui-ci, dir. V. Joly. ROLLAND, Amélie, Le journal de Jean Pichart, notaire royal et procureur au Parlement de Rennes (1589-1598), dir. P. HAMON. D. PICHOT. ussel, Bertrand, Michillimakinac : entre Français et Amérindiens, Coeur de l'alliance du Pays d'En-Haut (1715-1761), dir. P. HAMON. SILORET-RAULT, Martin, La nostalgie du futur : l'Unité populaire dans la presse d'opposition en Espagne. Les cas de Triunfo, Cuadernos para el dialogo et El Ciervo (1970-1978), dir. J. OBREGON ITURRA. TISSERAND, Guillaume, État des lieux historiographique, bibliographique et cartographique des fortifications de terre médiévales de Bretagne, dir. P.-Y. LAFFONT.

\section{Université de Tours}

\section{Thèses}

CHAMBRIER, Pauline, La réception de l'architecture au XVIe et XVIIe siècle par les contemporains : témoignages écrits et graphiques, dir. F. LEMERLE. CHARPY, Manuel, Le théâtre des objets. Espaces privés, culture matérielle et identité sociale à Paris, 1830-1914, dir. J.-L. PINOL. CHAUVET, Julie, Les Argiens et leurs dieux : espaces et temps sacrés, acteurs du culte et rites, dir. C. GRANDJEAN. CICOLANI, Veronica, Diffusion du mobilier de Goiasecca au nord des Alpes au premier âge du fer, dir. S. FICHTL. 
355 MEISS-EVEN, Marjorie, Être ou avoir. Les Ducs de Guise et leur paraître (1506-1588), dir. G. CHAIX,

\section{Master 2 (Recherche)}

ABAZA, Fouad, Un cas d'intégration de la pharmacologie grecque dans le monde arabe. La réception du De Materia Medica de Dioscoride dans le livre II du Canon de la Médecine d'Avicenne, dir. F.-O. TOUATI. 
CHEVReUIL, Charles, Le député Daniel Wilson et la politique de l'arrondissement de Loches (1877-1902), dir. R. BECK.

DELELIS, Cédric, Les lignes d'extrême droite en Indre-et Loire dans les années 1930, dir. R. BECK. DUBOST, Thomas, La percée du Front National à Dreux de 1978 à 1983, dir. J.-M. LARGEAUD.

GAVINI, Raphaël, Le journal « le Cri du Peuple de Paris », 1940-1944, dir. J.-M. LARGEAUD.

GUESDON, Emmanuelle, Les fêtes à Amboise au XVIIIe siècle (1700-1789), dir. U. KRAMPL.

IDOT, Bastien-Jacques, Turricella évêque de Marseille et confesseur de Marie de Médicis 1600-1618, dir. M. VERGÉ-FRANCESCHI.

LAMBERT, Mathieu, Le vignoble et les vignerons chinonais, du début du XIXe siècle à 1937. Naissance de la viticulture moderne, dir. R. BECK.

LAVRAT, Sandrine, Les pauvres et la pauvreté à Athènes à l'époque classique d'après les sources littéraires, dir. A. HELLER.

PEILLON, Maxime, Trajan et l'économie, dir. S. CROGIEZ-PETREQUIN.

PIVARD, Alix, L'armée d'Antigone le Borgne et la présence antigonique en Asie Mineure (321-301), dir. T. BOULAY.

SAVAUX, Antoine, La francophobie comme élément constitutif du nationalisme allemand 1770-1840, dir. R. BECK.

TROTIGNON, Estelle, Étude historique et architecturale de l'aitre Saint-Saturnin à Blois, dir. F.-O. TOUATI.

\section{Master 2 (« Alimentation »)}

BeLLIER, Néhémie, L'ananas, dir. M. de FERRIERE Le VAYer.

BERTHON, Simon, Les cantines du rail en France, dir. J.-P. WILLIOT.

BIRLIK, Bilge, Les restaurants turcs à Paris et en France depuis 1960, dir. J.-P. WiLLIoT.

CALOT, Laurie, Le vignoble de Bourgeuil 1850-1950, dir. M. de FERRIERE Le VAYER.

CHESNEAU, Anne Claude, Évolution du registre du vocabulaire des slogans publicitaires, dir. M. de FerRIERe Le VAyer.

ESTEP, Amber, Thanksgiving, Histoire d'une fête et d'une culture du repas de fête, dir. M. de FERRIERE LE VAYER.

GAUTHIER, Sophie, La salle à manger 1950-1960, dir. M. de FERRIERE LE VAYER.

LE Joncour, René, La cuisson à l'onde. Histoire d'une cuisson, dir. J.-P. WiLLIoT.

SENN, Anna Paula, Le petit déjeuner en France des années 1950 à nos jours, dir. J.P. WiLLIOT.

\section{Master 2 (Enseignement)}

CANIfET, Fanny, Les emblèmes du duc du Bery, dir. C. BousQuet.

HUVET, AXelle, Livret d'inauguration de la statue de Louis XV à Reims, dir. U. KRAMPL

MOREAU, Édouard Les représentations des victoires navales de Louis XIV, dir. M. VERGÉFRANCESCHI. 
PaUVRehomme, Maud, La place des femmes dans les publicités du registre alimentaire de 1950 à aujourd'hui, dir. J.-P. WiLLIot.

PHILIPPE, Marion, L'éducation militaire du prince à travers la littérature sous Charles viii, Louis xii et François $I^{\text {er }}$, dir. P. BRIOIST.

Rioland, Benoît, La représentation de l'slande au xvire siècle, pour Isaac la Peyrère, dir. P. BRIOIST.

SAFFRE, Aurore, Le temple d'Athéna Niképhoros, dir. T. BOULAY.

\section{Master 1 (Enseignement)}

CAILlaut, Dimitri, Historiographie de Bérenger de Tours, dir. B. Judic.

DELAC, Lucie, L'antisémitisme en Tourraine à la fin du xIxe siècle, dir. R. BECK.

FRESSART, Victor, Les fraudes alimentaire à Tours - 1930-1950, dir. J.-P. WILLIOT.

LAULANIE, Camille, L'Europe vu par un étudiant en 1945, dir. J.-M. LARGEAUD.

LE CHEVALIER Sandra, "Méchant démons ». Les démons babyloniens d'après la série d'exorcismes, dir. B. LIoN.

LUNEAU Jonathan, Les Francs Tireurs de Tours et la guerre de 1870-1871, dir. J.M. LARGEAUD.

\section{Master 2 (Archéologie)}

ANDRÉ, Élise, Les assemblages céramiques du HAD3 et de LT A1 des sites de Bourges, de la plaine de Voise.

CHoplin, Héléne, Marmoutier, Saint-Comes et la Loire, XIe-XVIIIe siècle : dynamique des berges et des îles, dir. E. LoRANS.

2 ConY, Alexandra, Les habitats ruraux à cours multiples au Second Âge de Fer en Gaule. Inventaire, typologie, cartographie, fonctions, dir. S. FIcHTL.

3 Descloux, Adeline, Les premiers édifices chrétiens en région Centre. Études de cas, dir. A. NISSEN-JAUBERT.

4 GENIES, Chloé, L'alimentation à Toursaux, XIe-XIIe siècles : ensemble faunique de la résidence des comtes d'Anjou, dir. M.-P. HORARD-HERBIN.

5 LEMAIRE, Pauline, L'insertion des thermes dans la ville gallo-romaine à partir de l'exemple de Javols-Architecture, dir. J. SEIGNE.

16 LuPu, Aurélien, Pratiques funéraires et représentation des genres au haut Moyen Âge, dir. A. NISSEN-JAUBERT.

7 SAUTEREAU, Aurélien, Les ensembles funéraires dans le département de l'Eure-et- Loir du IIIe au XIe siècle, dir. A. NISSEN-JAUBERT.

PAYSAn, Cathy, Les structures funéraires à poteaux porteurs du Second Âge du Fer dans la moitié Nord de la France, dir. S. FICHTL. 


\section{Master 1 (Archéologie)}

419 AVRILA, Raphaël, Étude archéologique de la charpente de la grange aux moines (Cne de Berthenay), dir. F. ÉPAuD.

FORGET, Cyprien, Inventaire des oppida dans l'Ouest de la Gaule aux IIe et Ier siècle avant J.-C., dir. S. FICHTL.

421 GERBAUD, Claire, La céramique du haut Moyen Âge de la fouille du cloitre de Saint martin de Tours, dir. P. HussI.

422 Jouteur, Noël, Essai de typo-chronologie des anses de vases en céramique de l'Artenar, dir. S. FICHTL.

LAMRISSI, Nissrine, Le mobilier lié au harnachement et au char de l'opidum de Bibracte, dir. S. FICHTL.

REIX, Morgane, Analyse architecturale de l'église Procope à Jerash (Jordanie), dir. J. SEIGNE.

RoLAND, Aude, Les épées avec fourreaux à bouterolles ajourés de forme circulaire ou sa variante losangée, dir. S. FICHTL.

INDEX

Thèmes : Angers, Brest, Lorient, La Rochelle, Le Mans, Nantes, Poitiers, Rennes, Tours 